\title{
Wiping Away Cellulitis: A Case of Factitious Disorder
}

\author{
Allison L. Wang, MD; Danielle J. Lospinoso, DO; Melissa M. Mauskar, MD
}

\section{PRACTICE POINTS}

- Consider exogenous factors or alternative diagnoses when a patient does not respond to appropriate care.

- Although dermoscopy is not used to diagnose cellulitis, it could be helpful in distinguishing cosmetic products used in dermatitis artefacta.

To the Editor:

Patients with psychocutaneous disorders present unique challenges to physicians. We illustrate the critical role that dermoscopy may play to illuminate exogenous skin pathology.

A 50-year-old woman with a reported medical history of systemic lupus erythematosus, chronic pain, and nonhealing leg ulcers presented to the emergency department with severe pain of the left lower leg and redness that was concerning for cellulitis. She sought treatment at an outside hospital for cellulitis 2 weeks prior but left against medical advice. Symptomatic review revealed chest pain, shortness of breath, nausea, vomiting, and diarrhea. The primary team started her on intravenous clindamycin and vancomycin for the presumed infection and scheduled narcotic medications due to concerns of intractable pain in the left leg. The dermatology department was consulted after failure to improve with 1 week of systemic antibiotics.

Physical examination revealed a geometric, atrophic, purple plaque on the left anterior shin from a prior leg ulcer as well as a diffuse red-pink patch extending from the knee to the ankle. Notably, the cellulitis spared the left posterior calf resting against the sheet and had a sharp line of demarcation at the distal shin. The leg was cool to the touch while the patient was distractible. She later reported that the leg was extremely tender to palpation. Dermoscopy revealed linear red pigments within skin furrows that accentuated skin lines (Figure). These findings raised suspicions of an external manipulation. The skin was wiped with an alcohol pad that removed a shimmering pink substance consistent in appearance to

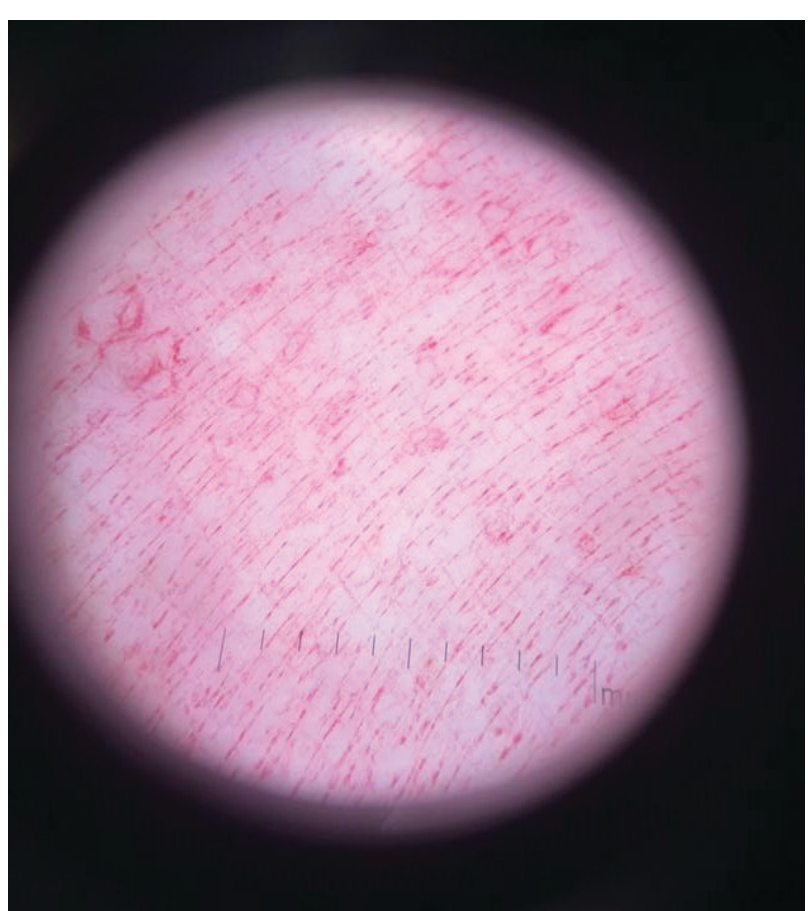

Dermoscopy of the affected area showed linear red pigments accentuating skin lines (original magnification $\times 10$ ).

Dr. Wang is from the Division of Dermatology, Cook County Health, Chicago, Illinois. Dr. Lospinoso is from San Antonio Uniformed Services Health Education Consortium, Texas. Dr. Mauskar is from the Department of Dermatology, University of Texas Southwestern Medical Center, Dallas. 
a cosmetic product. The skin beneath the cellulitis appeared normal.

On further review of the patient's medical record, it was noted that she was admitted several months ago for ulcers of the left leg. She had been to multiple hospitals and had numerous rounds of antibiotics. Biopsy of an ulcer revealed dermal fibrosis consistent with scarring. Aerobic bacteria, atypical mycobacteria, and fungal cultures were all negative. The physicians suspected a self-induced etiology consistent with dermatitis artefacta. The patient emphasized multiple psychosocial stressors as well as having frequent lupus flares despite repeated negative workup. Given the exaggerated symptoms and unnecessary hospital visits, she was given the diagnosis of factitious disorder (malingering or Munchausen syndrome). After extensive discussion, the patient was amenable to outpatient mental health counseling.
Dermoscopy is not a standard method to diagnose cellulitis of the skin; however, when patients present with an atypical response to appropriate care, the presumed diagnosis must be challenged. This patient had dramatized symptoms, false medical history, and numerous hospitalizations that were suspicious for factitious disorder. ${ }^{1}$ Furthermore, the physical examination was inconsistent with the classic course of cellulitis. In this case, dermoscopy had advantages over biopsies because it was noninvasive, gave immediate feedback, and provided a macroscopic view of the morphology. Via dermoscopy, we had an objective lens to distinguish cellulitis from cosmetic product and to obtain the correct diagnosis.

\section{REFERENCE}

1. Harth W, Taube KM, Gieler U, Facticious disorders in dermatology. J Dtsch Dermatol Ges. 2010;8:361-372. 